\title{
Considerations before the application of 5- hydroxymethylation levels of long non-coding RNAs for non-invasive cancer diagnosis
}

\author{
Zhou Zhang', Chang Zeng ${ }^{1}$, Wei Zhang ${ }^{1,2}$ \\ 'Department of Preventive Medicine, Northwestern University Feinberg School of Medicine, Chicago, Illinois 60611, USA. \\ ${ }^{2}$ The Robert H. Lurie Comprehensive Cancer Center, Northwestern University Feinberg School of Medicine, Chicago, Illinois \\ 60611, USA.
}

Correspondence to: Prof. Wei Zhang, Department of Preventive Medicine, Northwestern University Feinberg School of Medicine, 680 N. Lake Shore Dr., Suite 1520, Chicago, Illinois 60611, USA. E-mail: wei.zhang1@northwestern.edu

How to cite this article: Zhang Z, Zeng C, Zhang W. Considerations before the application of 5-hydroxymethylation levels of long non-coding RNAs for non-invasive cancer diagnosis. Extracell Vesicles Circ Nucleic Acids 2022;3:10-3.

https://dx.doi.org/10.20517/evcna.2021.22

Received: 29 Nov 2021 First Decision: 22 Dec 2021 Revised: 10 Jan 2022 Accepted: 19 Jan 2022 Published: 21 Jan 2022

Academic Editors: Yoke Peng Loh, Erik A. Sistermans Copy Editor: Yue-Yue Zhang Production Editor: Yue-Yue Zhang

\begin{abstract}
Previous studies have suggested that aberrant 5-hydroxymethylcytosines (5hmC) modifications are related to cancer pathobiology. Genome-wide profiling $5 \mathrm{hmC}$ in circulating cell-free DNA (cfDNA) using the highly sensitive chemical labeling-based $5 \mathrm{hmC}$-Seal technique has been demonstrated to have the potential to be a robust epigenomic tool for cancer biomarker discovery. Prior studies have mostly focused on cfDNA-derived 5hmC-Seal data summarized in well-annotated genic features (e.g., gene bodies) or unbiased bins. Zhou et al. recently proposed long non-coding RNAs (IncRNAs) as an alternative molecular target for biomarker discovery using publicly available $5 \mathrm{hmC}$-Seal data. Considering its potential clinical impact, we would like to comment on Zhou et al. and advocate more serious consideration of critical issues such as the availability of clinical information and technical variables, especially when performing secondary analysis using publicly available data, with the aim of improving data transparency and translatability.
\end{abstract}

Keywords: 5-Hydroxymethylcytosine, long non-coding RNA, cell-free DNA, cancer biomarker

The 5-hydroxymethylcytosine $(5 \mathrm{hmC})$ is an emerging epigenetic marker that reflects gene activation

(c) The Author(s) 2022. Open Access This article is licensed under a Creative Commons Attribution 4.0

International License (https://creativecommons.org/licenses/by/4.0/), which permits unrestricted use, sharing, adaptation, distribution and reproduction in any medium or format, for any purpose, even commercially, as long as you give appropriate credit to the original author(s) and the source, provide a link to the Creative Commons license, and indicate if changes were made. 
status ${ }^{[1]}$. Previous studies have suggested that aberrant $5 \mathrm{hmC}$ modifications are related to cancer pathobiology. Genome-wide profiling of $5 \mathrm{hmC}$ in circulating cell-free DNA (cfDNA) using the 5hmC-Seal technique $^{[2]}$, a highly sensitive chemical labeling approach suitable for a very limited amount of clinical biospecimens (e.g., 1-2 ng of cfDNA from a few $\mathrm{mL}$ of plasma) has been demonstrated by our team and other groups to be a robust epigenomic tool for cancer biomarker discovery with the goal of achieving noninvasive cancer diagnosis and prognosis ${ }^{[3-6]}$.

Analytically, although our previous studies mostly focused on the $5 \mathrm{hmC}$-Seal profiles summarized in wellannotated genic features (e.g., gene bodies) or unbiased bins, recently, we started exploring the possibility of integrating $5 \mathrm{hmC}$ profiles summarized for long non-coding RNAs (lncRNAs) and repetitive elements to improve biomarker discovery using glioblastoma (GBM) as an example ${ }^{[3]}$. Specifically, in the cell, lncRNAs are known to regulate gene expressions at both transcriptional and post-transcriptional levels, and play important and heterogeneous regulatory roles in nearly all cellular and biological processes, including transcriptions, translation, and nuclear trafficking, as well as tumorigenesis and therapy resistance ${ }^{[7]}$. In GBM, dysregulation of lncRNAs can contribute to the epithelial-mesenchymal transition, therefore promoting cancer metastasis ${ }^{[8]}$. In addition, a recent study reported a positive association between $5 \mathrm{hmC}$ and $\operatorname{lncRNA}$ transcription in colorectal cancer, indicating the regulatory role of $5 \mathrm{hmC}$ on lncRNA expression $^{[9]}$. Given its tissue-specificity and roles in tumor initiation, progression and resistance to therapy, lncRNAs remain to be promising markers for cancer diagnosis and prognosis.

Specifically, we read with interest that a recent study published by Zhou et al. ${ }^{[10]}$ described the development of plasma-derived 5hmC-LncRNA diagnostic score (5hLD-score) for cancer diagnosis and surveillance using publicly available $5 \mathrm{hmC}$ data. The proposed $5 \mathrm{hLD}$-score was shown the capability of distinguishing tumors from healthy controls in their training and internal validation cohorts. Further validation showed the $5 \mathrm{hLD}$-score achieved area under the curve (AUC) of 0.85, 0.89, and 0.77 in a non-small cell lung cancer cohort, an esophageal cancer cohort, and a hepatocellular carcinoma (HCC) cohort, respectively. The authors identified an association between the 5hLD-score and the progression of liver cancer in the HCC cohort, as well as the capability to identify the origin and location of tumors. This study further supported the clinical potential of $5 \mathrm{hmC}$ levels in lncRNAs for cancer early detection and progression monitoring. However, we would like to comment on a few important issues of Zhou et al. and advocate that there are several critical issues that need to be taken into consideration in order to make an informed conclusion of the current status of applying $5 \mathrm{hmC}$ levels in lncRNAs as a marker for cancer diagnosis and prognosis, especially when such a conclusion was drawn from performing secondary data analysis using public data.

Firstly, during statistical modeling, differential $5 \mathrm{hmC}$ modifications should be identified in the training set solely. Instead, Zhou et al. used the whole Li's cohort (training and internal validation set combined) to perform the differential analysis. This procedure would have caused data leakage, which introduced the knowledge of the validation set into the modeling process, and could have led to model overfitting in the validation set. Therefore, the observed differences in terms of the AUCs between the internal validation set and the independent validation set presented by Zhou et al. could be due to data leakage and model overfitting, which should be evaluated using appropriate tests such as the Delong test ${ }^{[11]}$.

Secondly, when using the $5 \mathrm{hmC}$ profiles generated from different platforms/protocols, sequencing length, depth, or platform information should be taken into considerations. Regarding these potential technical biases, Zhou et al. did not take them into considerations in their analysis. To our best knowledge, the Li's cohorr $^{[6]}$ was sequenced with 150 base-pair (bp) paired-end library, while the Cai's liver cancer cohort ${ }^{[5]}$ was sequenced with $38 \mathrm{bp}$ paired-end library. In addition, the publicly available $5 \mathrm{hmC}$ data were generated at 
different times and core facilities. Those unaccounted factors, taken together, could cause substantial batch effects, with the likelihood of leading to misinterpretation of the results.

Thirdly, clinical variables, such as age, gender, tumor stages, place of residence, and lifestyle, have been established as potential confounders in epigenetic studies. These variables (known or hidden) contribute to the epigenetic differences between cases and controls. Not appropriately adjusting for these confounding variables could lead to biased interpretation of results. For examples, in figure 5, Zhou et al. ${ }^{[10]}$ argued that the 5hLD-scores were associated with liver cancer progression. However, this finding could be confounded by patient's age, as the liver cancer patients were much older than patients with hepatitis B infection history in the Cai cohort ${ }^{[4]}$.

Finally, unlike mRNAs with protein-coding potential or microRNAs with high sequence conservations, $\operatorname{lncRNAs~possessing~unique~features~such~as~lower~transcription~rate,~reduced~stability~and~lower~expression~}$ levels can pose analytic constraints in the characterization and annotation of $\operatorname{lncRNAs^{[12]}}$. For example, the GENCODE $^{[13]} \operatorname{lncRNAs}$ were identified from RNA-Seq data and algorithm not optimized for the full exploitation and annotation for non-polyA lncRNA transcripts or functional lncRNAs with relatively lower expression. Furthermore, given the relatively lower expression of lncRNA in non-brain tissue types, the signal to noise ratios of $5 \mathrm{hmC}$ mapping over lncRNA regions on cfDNA are expected to be even lower in non-brain cancer patients included at least in theory. However, Zhou et al. did not provide any evaluation of the expression levels or tissue-specificity of these lncRNAs before proceeding to the marker discovery phase. As a result, the $5 \mathrm{hmC}$ profiles of $\mathrm{lncRNAs}$ in the current study could have been subjected to random noise due to low abundance. Last but not least, we observed synergistic effects between the $5 \mathrm{hmC}$ of IncRNAs and other genomic feature types (i.e., gene body, repetitive elements and histone marks) in our GBM study ${ }^{[3]}$, it would be interesting if future studies could incorporate other genomic feature types and compare the performance by feature type, separately and integratively.

In conclusion, in our opinion, the $5 \mathrm{hmC}$ levels of $\ln \mathrm{R} R \mathrm{NAs}$ could be a promising biomarker for cancer diagnosis and monitoring, though future large studies of individuals with more comprehensive clinical, pathological, and epidemiological information, as well as the application of more robust data analysis plans (e.g., consideration of hidden variables) will help improve data transparency and provide more insights into the translatability of these molecular targets.

\section{DECLARATIONS}

\section{Authors' contributions}

Made substantial contributions to conception: Zhang Z, Zhang W

Drafted the manuscript: Zhang Z, Zeng C, Zhang W

Approved the final version: Zhang Z, Zeng C, Zhang W

\section{Availability of data and materials}

Not applicable.

\section{Financial support and sponsorship}

This work was supported in part by National Institutes of Health grants: R01CA223662, U01CA217078, R21CA209345, and R21CA187869.

\section{Conflicts of interest}

Zhang W has been an advisor to Epican Genetech, which has a license of the $5 \mathrm{hmC}$-Seal technique from the University of Chicago for clinical application. Other authors declared that there are no conflicts of interest. 


\section{Ethical approval and consent to participate}

Not applicable.

\section{Consent for publication}

Not applicable.

\section{Copyright}

(C) The Author(s) 2022.

\section{REFERENCES}

1. Branco MR, Ficz G, Reik W. Uncovering the role of 5-hydroxymethylcytosine in the epigenome. Nat Rev Genet 2011;13:7-13. DOI PubMed

2. Song CX, Szulwach KE, Fu Y, et al. Selective chemical labeling reveals the genome-wide distribution of 5-hydroxymethylcytosine. Nat Biotechnol 2011;29:68-72. DOI PubMed PMC

3. Cai J, Zeng C, Hua W, et al. An integrative analysis of genome-wide 5-hydroxymethylcytosines in circulating cell-free DNA detects noninvasive diagnostic markers for gliomas. Neurooncol Adv 2021;3:vdab049. DOI PubMed PMC

4. Chiu BC, Zhang Z, You Q, et al. Prognostic implications of 5-hydroxymethylcytosines from circulating cell-free DNA in diffuse large B-cell lymphoma. Blood Adv 2019;3:2790-9. DOI PubMed PMC

5. Cai J, Chen L, Zhang Z, et al. Genome-wide mapping of 5-hydroxymethylcytosines in circulating cell-free DNA as a non-invasive approach for early detection of hepatocellular carcinoma. Gut 2019;68:2195-205. DOI PubMed PMC

6. Li W, Zhang X, Lu X, et al. 5-Hydroxymethylcytosine signatures in circulating cell-free DNA as diagnostic biomarkers for human cancers. Cell Res 2017;27:1243-57. DOI PubMed PMC

7. Uszczynska-Ratajczak B, Lagarde J, Frankish A, Guigó R, Johnson R. Towards a complete map of the human long non-coding RNA transcriptome. Nat Rev Genet 2018;19:535-48. DOI PubMed PMC

8. Kiang KM, Zhang XQ, Leung GK. Long non-coding RNAs: the key players in glioma pathogenesis. Cancers (Basel) 2015; 7:1406-24. DOI PubMed PMC

9. $\mathrm{Hu} \mathrm{H}$, Shu M, He L, et al. Epigenomic landscape of 5-hydroxymethylcytosine reveals its transcriptional regulation of lncRNAs in colorectal cancer. Br J Cancer 2017;116:658-68. DOI PubMed PMC

10. Zhou M, Hou P, Yan C, et al. Cell-free DNA 5-hydroxymethylcytosine profiles of long non-coding RNA genes enable early detection and progression monitoring of human cancers. Clin Epigenetics 2021;13:197. DOI PubMed PMC

11. DeLong ER, DeLong DM, Clarke-Pearson DL. Comparing the areas under two or more correlated receiver operating characteristic curves: a nonparametric approach. Biometrics 1988;44:837-45. PubMed

12. Cao H, Wahlestedt C, Kapranov P. Strategies to annotate and characterize long noncoding RNAs: advantages and pitfalls. Trends Genet 2018;34:704-21. DOI PubMed

13. Aken BL, Ayling S, Barrell D, et al. The Ensembl gene annotation system. Database (Oxford) 2016;2016:baw093. DOI PubMed $\mathrm{PMC}$ 